\title{
Mecanismos de evasión del sistema del complemento utilizados por Trypanosomacruzi
}

\author{
Valentina Tapia ${ }^{1}$ Lic. MV, Pablo Galdames ${ }^{1}$ Lic. MV, Galia Ramírez T. ${ }^{1}$ MV, PhD \\ ${ }^{1}$ Laboratorio de Parasitología, Departamento de Medicina Preventiva Animal. Facultad de Ciencias \\ Veterinarias y Pecuarias. Universidad de Chile. Santa Rosa 11.735, La Pintana. Casilla 2, correo 15, La \\ Granja. Email: galiaram@uchile.cl
}

\begin{abstract}
Resumen
Trypanosomacruzi es el agente causal de la enfermedad de Chagas, la infección parasitaria más importante de Latinoamérica. Se considera una enfermedad crónica, que causa discapacidad, para la cual no existe tratamiento efectivo. Para poder establecer una infección de manera exitosa, T. cruzi cuenta con múltiples mecanismos para evadir la respuesta inmune del hospedero. Entre estos múltiples mecanismos, los tripomastigotes, formas infectantes del parásito, cuentan con mecanismos que los hacen resistir el ataque del sistema del complemento. Por el contrario, los epimastigotes son altamente susceptibles. Para resistir la lisis mediada por el complemento, T. cruziutilizadiversos factores que interfieren con las funciones de la ruta clásica o alterna en distintos puntos de la cascada. Sin embargo, estos mecanismos no son del todo comprendidos. Entre las proteínas que participan en la resistencia a la lisis están el factor acelerador del decaimiento de las convertasas de $T$. cruzi(T-DAF), la proteína reguladora del complemento de $T$. cruzi (CRP), calreticulina de T. cruzi(TcCRT), factor H, entre otras. A continuación revisaremos el rol de estas proteínas en la evasión del sistema del complemento del hospedero por T. cruzi.
\end{abstract}

Palabras clave: Trypanosomacruzi, Proteinas reguladoras del complemento (CRPs), factor acelerador del decaimiento de tripomastigotes (T-DAF), Factor H (FH).

\section{Introducción}

Trypanosomacruzi es el agente causal de la enfermedad de Chagas, la infección parasitaria transmitida por vectores más importante de Latinoamérica, donde se estima que aproximadamente 15 millones de personas se encuentran infectadas (Coura and Dias, 2009). Sin vacuna o tratamiento apropiado disponible, el principal control se basa en la prevención de la transmisión vectorial a través de la eliminación del vector dentro de las viviendas.

Recientemente, la Organización Mundial de la Salud ha realizado una lista con más de 180 mamíferos domésticos y peridomésticos que participan como importantes reservorios (Haberland et al., 2012).

T. cruzi es transmitido por hemípteros de la familia Reduviidae, los cuales portan al parásito en su intestino (Macedo et al., 2002). Durante la succión de sangre, el insecto deposita sus deyecciones (mezcla de orina y heces) en la piel del individuo donde se encuentran las formas tripomastigotesmetacíclicos infectantes, los cuales ingresan en el hospedero mamífero por mecanismos aún no bien dilucidados.

Una vez dentro de la célula mamífera, los tripomastigotes son recluidos dentro de un compartimento denominado fagosoma o vacuola parasitófora, desde donde, eventualmente, escapan para 
diferenciarse en amastigotes (Mortara et al., 2005) y multiplicarse intracelularmente. Éstos pasan a su estado tripomastigote sanguíneo y son liberados, junto con algunos amastigotes, durante la ruptura celular. Los amastigotes libres infectan nuevas células y los tripomastigotes re-ingresan al torrente sanguíneo. El insecto adquiere las formas tripomastigotes por ingestade sangre infectada. En el estómago del insecto, los tripomastigotes se transforman en dos estados replicativos: epimastigotes y esferomastigotes. Estos proliferan por división celular $\mathrm{y}$, una vez que llegan al recto, se diferencian en tripomastigotesmetacíclicos infectantes, para completar el ciclo (Macedo et al., 2002).

La enfermedad de Chagas es considerada una compleja zoonosis, que en humanos puede producir una infección crónica caracterizada principalmente por la producción de megasíndromes como el megacolon y megaesófago, y la cardiomiopatía chagásica crónica (CCC).

A pesar de las exitosas iniciativas multinacionales para interrumpir la transmisión vectorial de la enfermedad en Latinoamérica, se estima que existen aún 28 millones de personas en riesgo de adquirir la infección y aproximadamente, 15 millones de personas infectadas, con una incidencia anual y mortalidad de 41.200 y 12.500 personas, respectivamente (Haberland et al., 2012).

La evasión del sistema inmune es clavepara la sobrevida de los patógenos dentro de su hospedero. Por esta razón, no es sorprendente que la co-existencia y la co-evolución de humanos y microorganismos haya producido una multitud de mecanismos de origen microbiano para atenuar o escapar de este ataque (Lambris et al., 2008). Durante la fase aguda de la infección chagásica, existe un control inmunológico dado por la respuesta inmune humoral y celular. Sin embargo, esta respuesta disminuye con el tiempo, y se suma a la liberación de elementos de supresión de la respuesta inmune por parte del parásito. Esto lleva a un ineficiente control del parásito por este sistema y una incompleta erradicación de éste desde los tejidos, generando una persistencia parasitaria y cronicidad (Haberland et al., 2012). Para el caso de T. cruzi, tanto los tripomastigotes sanguíneos como de cultivo son resistentes a la lisis mediada por el sistema del complemento, importante brazo efector que participa tanto en la inmunidad innata como adaptativa (Kipnis et al., 1981; Nogueira et al., 1975), a diferencia de los epimastigotes que son sensibles. Para resistir la lisis del complemento, los tripomastigotes de $T$. cruzi producen factores reguladores que interfieren con las funciones de la ruta clásica o alterna en distintos niveles de la cascada del complemento.

El sistema del complemento es un potente mecanismo inmune, conformado por proteínas solubles, proteasas y receptores unidos a la membrana, encargados de la identificación y eliminación de microorganismos invasores y detritus celular. La cascada del complemento es activada a través de 3 distintas vías: clásica, alterna y de las lectinas (Beucher and Norris, 2008). La actividad del complemento es controlada por proteínas reguladoras que protegen a las células y tejidos del hospedero del daño mediado por la activación del sistema (Hourcade et al., 1989). Algunas de ellas inhiben el ensamblaje de las convertasas, a través de la aceleración del decaimiento de las convertasas, sirven como cofactores enzimáticos(Kemper et al., 2009), se unen a proteínas del sistema del complemento inhibiendo su función, entre otras propiedades que serán discutidas más adelante.

\section{Sistema del complemento}

El sistema del complemento es un componente central de la respuesta inmune innata y cumple numerosas funciones, incluyendo el reconocimiento de células extrañas, comunicación con la respuesta inmune adaptativa y su activación, y la remoción de restos celulares. Está compuesto por una red de proteínas que circulan por el torrente sanguíneo en forma soluble o unidas a la superficie celular, las cuales sirven de sustratos, enzimas o moduladores en una cascada de activación proteolítica extracelular (Lambris et al., 2008). Actualmente, se le conocen 3 rutas de activación: clásica, alterna y de las lectinas. La activación de cualquiera de ellas lleva a la formación de complejos enzimáticos cruciales (convertasas de $\mathrm{C} 3$ y C5), opsonización de células o moléculas blanco con $\mathrm{C} 3 \mathrm{~b} / \mathrm{iC} 3 \mathrm{~b}$ (seguido de fagocitosis), liberación de anafilotoxinas pro-inflamatorias $(\mathrm{C} 4 \mathrm{a}, \mathrm{C} 5 \mathrm{a}, \mathrm{C} 3 \mathrm{a})$ que atraen otras células del sistema inmune y finalmente, la 
formación de un complejo de ataque a membrana o CAM, que media la lisis osmótica de la célula blanco (Sjoberg et al., 2009).

\subsection{Rutas de activación del sistema del complemento}

\subsubsection{La ruta clásica}

Es estimulada por el reconocimiento de complejos antígeno- anticuerpopor la proteína $\mathrm{Clq}$, en una superficie celular extraña(Lambris et al., 2008). Luego, C1q interacciona con dos pares de subunidades catalíticas denominadas C1r y C1s (Arlaud et al., 2002; Lepow et al., 1963), formando el complejo C1. C1r digiere y activa a $\mathrm{C} 1 \mathrm{~s}$,llevando a la activación proteolítica de $\mathrm{C} 4$ y $\mathrm{C} 2$ para formar la convertasade $\mathrm{C} 3$ de la ruta clásica(C4b2b)(Wallis et al., 2010).

\subsubsection{La ruta de las lectinas}

A diferencia de la ruta clásica, la ruta de las lectinas es independiente de la presencia de anticuerpos y es estimulada por el reconocimiento, por parte de MBL (mannose-bindinglectin) oficolinas, de carbohidratos que les sirven de ligando y que están presentes en la superficie de muchos agentes microbianos(Lambris et al., 2008). MBL y ficolinas se asemejan estructuralmente a C1q y se unen a homólogos de C1r y C1s llamadas serino-proteasas asociadas a MBL (MASPs) (Wallis et al., 2010). El resto de la ruta ocurre de manera similar a la ruta clásica.

\subsubsection{La ruta alterna}

A diferencia de las otras vías, la ruta alterna es estimulada por hidrólisis espontánea de C3 nativo o la presencia de estructuras de superficie extrañas, en ausencia de una molécula central de reconocimiento (Lambris et al., 2008). La vía es iniciada por la autoactivación deC3 inestable, a través de la unión de properdina o como una ruta de amplificación para las otras dos rutas, generándose C3b(Sjoberg et al., 2009). A pesar de esta auto-activación, la ruta alterna se encuentra finamenteregulada por proteínas de control del complemento que serán discutidas más adelante.

\subsubsection{Formación del complejo de ataque a la membrana (CAM)}

Aun cuando las diferentes rutas de activación del complemento son activadas por un grupo distinto de moléculas, todo culmina en la formación de las convertasas, las principales enzimas de la cascada, y la generación de la opsonina C3b. Las C3 convertasasde la ruta clásica $(\mathrm{C} 4 \mathrm{~b} 2 \mathrm{~b})$ o de la ruta alterna $(\mathrm{C} 3 \mathrm{bBb})$ digieren y activan a $\mathrm{C} 3$, generando los fragmentos $\mathrm{C} 3 \mathrm{a}$ y $\mathrm{C} 3 \mathrm{~b}$. Este proceso expone en los fragmentos $\mathrm{C} 3 \mathrm{~b}$ un sitio tiol-éster altamente reactivo que puede formar un enlace covalente con una superficie blanco cercana, iniciando el ensamblaje de una C3convertasa adicional (ruta alterna de amplificación). C3b tambiénpuede unirse directamente a la C3 convertasa, para formar la C5 convertasa (C4b2b3b ó C4bBb3b) la cual digiere y activa a C5, para producir los fragmentos $\mathrm{C} 5 \mathrm{a}$ y $\mathrm{C} 5 \mathrm{~b}$. La formación del CAM comienza en fase fluida con la asociación de $\mathrm{C} 5 \mathrm{~b}$ con los componentes $\mathrm{C} 6$ y $\mathrm{C} 7$. El complejo resultante $\mathrm{C} 5 \mathrm{bC} 6 \mathrm{C} 7$ se inserta en la membrana microbiana donde se une a $\mathrm{C} 8$ y se completa con la polimerización de C9. El CAM completo promueve la ruptura de la membrana y la lisis celular (Kemper et al., 2010).

\subsection{Proteínas de control del complemento}

El sistema del complemento utiliza un complejo grupo de proteínas de control para seleccionar sus blancos y regular la amplificación a travésdefragmentos de unión a C3. Diferentes proteínas reguladoras,propias del hospedero, se unen a las células ylas protegen de la citólisis mediada por la activación del sistema del complemento(Liszewski and Atkinson, 1996; Pangburn, 2000).Es así como C3b y C4b son rápidamente inactivadas por la proteólisis en fragmentos producida por la serino-proteasa factor I en presencia de co-factores como la proteína co-factora de membrana (MCP) y el receptor del complemento 1 (CR1) que se encuentran unidos a la membrana, además de factor $\mathrm{H}(\mathrm{FH})$, el cual está unido a la superficie del hospedero. Además, el inhibidor de $\mathrm{C} 1$ (C1-INH) inactiva C1r, C1s y MASP2 (Davis et al., 2008). La activación es también regulada a través de la prevención del ensamblaje de la $\mathrm{C} 3$ convertasa o una 
vez que ésta está formada, por la inhibición de su actividad, debido a la acción de factores aceleradores del decaimiento como DAF (CD55), la proteína de unión a C4 (C4-bp), FH y la proteína de unión a membrana, encontrada solo en ratones, Crry. Además, la formación del CAM es negativamente regulada por la proteína $\mathrm{S}$ (glicoproteína plasmática sintetizada por los endoteliocitos), vimentina (una proteína del citoesqueleto) y CD59, que interfieren con el ensamblaje del CAM (Huang et al., 2006).

\section{Inhibición del sistema del complemento por T. cruzi}

El Para establecer una infección exitosa, las formas infectantes de T. cruzi, los tripomastigotes deben evadir al sistema inmune. Se han descrito numerosos mecanismos de evasión. Entre ellos, podemos destacar los utilizados por el parásito para evadir al sistema del complemento del hospedero.

A continuación mencionaremos las proteínas descritas para T. cruzi relacionadas con la evasión de este sistema y su función:

\subsection{Proteína reguladora del complemento de T. cruzi (CRP)}

CRP es una proteína con función reguladora del complemento que se encuentra anclada a la membrana del parásito, a través de un tallo de glico-fosfatidilinositol (GPI).Se encuentra presente en el flagelo y bolsillo flagelar (Van Voorhis et al., 1993). Funcionalmente, es similar a la proteína DAF de humanos, pero carece de identidad o similitud de secuencias con DAF y otras CRPs de mamíferos(Beucher and Norris, 2008; Norris et al., 1991). Esta glicoproteína de 160 kDase une no covalentemente a $\mathrm{C} 3 \mathrm{~b}$ y $\mathrm{C} 4 \mathrm{~b}$, inhibiendo el ensamblaje de la convertasa de $\mathrm{C} 3$ proteolíticamente activa, contribuyendo así a la inhibición de la lisis del sistema del complemento sobre la superficie parasitaria (Beucher et al., 2003; Norris et al., 1991; Norris and Schrimpf, 1994). Es expresada por tripomastigotes, pero no por epimastigotes, sin embargo, cuando se transfectanepimastigotes para que expresen esta proteína, estos son capaces de evitar la lisis mediada por el sistema del complemento(Norris, 1998).

El genoma de T. cruzi contiene múltiples copias de crp y las proteínas codificadas por estos genes comparten similitud de secuencias con miembros de la superfamilia de las trans-sialidasas (TS) de T. cruzi. Dentro de esta familia, las CRPs forman una de las 3 distintas subfamilias funcionales que carecen de actividad TS(Cross and Takle, 1993) y es funcionalmente similar a las proteínas microbianas y mamíferas que regulan la activación del complemento(Osorio et al., 2012).

\subsection{Factor acelerador del decaimiento detripomastigotes de T. cruzi (T-DAF)}

T-DAF es una glicoproteína de membrana de 87 a 93 $\mathrm{kDa}$ (Osorio et al., 2012). Su cDNA comparte similitud con DAF de humanos e inhibe la lisis parasitaria (Tambourgi et al., 1993). Posee una amplia distribución celular y está anclada a la membrana plasmática a través de un tallo GPI (Davitz et al., 1986). La proteína está presente en las formas tripomastigotetanto sanguíneo, como de cultivo y metacíclico(Tambourgi et al., 1995). Se une a C3b y $\mathrm{C} 4 \mathrm{~b}$, regulando tanto la ruta clásica como la alterna, donde altera o destruye, no permanentemente, los componentes del complemento (Kinoshita et al., 1986). DAF-1 inhibe el ensamblaje y acelera el rápido decaimiento de la C3 y C5 convertasa de la ruta clásica y alterna del sistema del complemento(Solana et al., 2012).

\subsection{Proteína inhibidora del receptor C2 del complemento tri-funcional (CRIT)}

Proteína expresada por tripomastigotes que inhibe la activación de la ruta de las lectinas del complemento. Algunos péptidos derivados de la proteína inhiben la unión de $\mathrm{C} 2$ a la superficie parasitaria y la muerte del parásito. CRIT es una molécula que se expresa en la superficie de los tripomastigotesmetacíclicos(Cestari Idos et al., 2008). Su dominio 1 extracelular inhibe la digestión de C2 por MASP2 y deteriora la formación de la convertasade C3 (Cestari Idos et al., 2009). Los parásitos que sobre expresan CRIT son altamente resistentes a la lisis mediada por el complemento. La 
sobre expresión del gen crit en epimastigotes, la forma no infectante del parásito y altamente susceptible al sistema del complemento, les confiere resistencia (Cestari Idos et al., 2008). Sin embargo, la capacidad de resistir a las diferentes rutas del complemento varía entre distintas cepas de $T$. cruzi;la cepa $\mathrm{Y}$ es más resistente a la ruta alterna y de las lectinas, que la cepa Colombiana(Osorio et al., 2012).

CRIT es una proteína de trans-membrana que presenta un dominio extracelular N-terminal denominado ed1. Este dominio tiene similitud con la cadena $\mathrm{C} 4 \beta$, involucrada en la unión a C2 (Cestari Idos et al., 2009; Inal and Schifferli, 2002). El dominio ed1se une a $\mathrm{C} 2 \mathrm{e}$ inhibe la digestión de C1s y MASP2, previniendo la formación de la convertasa de C3 de la ruta clásica y de las lectinas(Cestari et al., 2012; Cestari Idos et al., 2009; Inal, 2005; Inal and Schifferli, 2002).

\subsection{Glicoproteína 58/68 (gp 58/68)}

Glicoproteína de 58 a 68 kDa, receptor de colágeno/fibronectina de T. cruzi(Fischer et al., 1988), que se expresa sólo en las formas tripomastigotas(Velge et al., 1988). La proteína inhibe la formación de la convertasa de $\mathrm{C} 3$ de la ruta alterna, tanto en la superficie celular como en fase fluida, contribuyendo a la inhibición de la lisis mediada por complemento. Este efecto inhibitorio dependería, en mayor medida, de su interacción con el factor B (Fischer et al., 1988). Gp58/68 purificada inhibe la formación de la convertasa de $\mathrm{C} 3$ de la ruta alterna,tanto en fase fluida como unida a la superficie, de manera dosis dependiente. A diferencia de DAF, gp $58 / 68$ no disocia la convertasa de C3. Es por esto que la inhibición de la convertasa parece depender de su disociación por $\mathrm{fB}$, más que de $\mathrm{C} 3 \mathrm{~b}$ (Fischer et al., 1988).

\subsection{Calreticulina de T. cruzi (TcCRT)}

Proteína presente en el interior y en la superficie de tripomastigotes de $T$. cruzi, donde se une a C1q, MBL y ficolinas, de la ruta clásica y de las lectinas del sistema del complemento(Ferreira et al., 2004; Valck et al., 2010). Esta unión genera una inactivación de la ruta clásica en su etapa inicial, inhibiendo la formación del complejo de ataque a membrana (Ferreira et al., 2004).
Esta misma función se ha descrito también, más recientemente, para CRT de otro tripanosomátido, comoTrypanosomacarassii, parásito extracelular, de gran importancia económica en peces. CRT de $T$. carassii se une a $\mathrm{C} 1 \mathrm{q}$ inhibiendo la ruta clásica del sistema del complemento (Oladiran and Belosevic, 2009), de manera muy similar a la descrita para TcCRT(Ferreira et al., 2004). Enotros parásitos nematodos como Haemonchuscontortus, también se ha descrito la unión de CRT, presente en moléculas de excreción/secreción de los parásitos adultos, a proteínas del sistema del complemento del hospedero como la proteína C-reactiva (Naresha et al., 2009; Suchitra et al., 2008; Suchitra and Joshi, 2005).

Por otro lado, TcCRTpresente en la membrana de los tripomastigotes de $T$. cruzi actúa como un factor de virulencia(Johnson et al., 2012; Ramirez et al., 2011), aumentando la capacidad infectiva del parásito mediada por C1q (Ramirez et al., 2012; Ramirez et al., 2011; Rimoldi et al., 1989).

\subsection{Factor $H(F H)$}

FH es una proteína reguladora negativa del sistema del complemento, responsable del reconocimiento de las células hospederas y la discriminación entre patógenos microbianos durante la activación de la ruta alterna (Pangburn, 2000). Es una molécula de 20 sitios de consenso (SCR) que forman una cadena flexible, conformando una variedad de sitios funcionales para interactuar con proteínas del complemento y marcadores de superficie. Cada uno de estos 20 dominios está formado por 60 aminoácidos. Estos SCR poseen numerosos sitios funcionales (Figura 1). Factor $\mathrm{H}$ posee 3 sitos de unión a C3b. El situado en SCR 4 es el responsable de la actividad aceleradora del decaimiento de la convertasa de C3 y C5 de la ruta alterna, además de ser cofactor de factor I, una serinoproteasa que inactiva a $\mathrm{C} 3 \mathrm{~b}$. Los otros 2 sitios de unión a C3b no poseen actividad reguladora directa sobre el sistema del complemento. Los otros sitios interactúan con polianiones de varios tipos. El primero de estos está localizado en el SCR 7, el segundo cerca de SCR 13 y el último, en SCR 18-20, queinteractúa con ácido siálico(Pangburn, 2000).

Algunas partículas biológicas, incluyendo muchos tejidos del hospedero, carecen de estas proteínas 
reguladores. Este hecho las convierte en activadoras de la ruta alterna del sistema del complemento en su superficie dependiendo del tipo y densidad de marcadores en su superficie capaces de ser reconocidos por FH (Pangburn, 2000). FH en la superficie, al igual que DAF, MCP y CR1 en las células propias, controla si el $\mathrm{C} 3 \mathrm{~b}$ inicial es amplificado o rápidamente inactivado. La mayoría de los microrganismos son fuertes activadores de la ruta alterna del sistema del complemento. Muchos de estos patógenos han adquirido un mecanismo de mimetización utilizando marcadores del hospedero y utilizando el mismo sistema de sitios de reconocimiento para FH que en el hospedero(Pangburn, 2000). Es así, como en el caso de T. cruzi, el ácido siálico juega un papel importante en el proceso infeccioso, sin embargo, como el parásito no es capaz de sintetizarlo, éste expresa una particular enzimatransialidasa (TcTS), que transfiere el ácido siálico desde sus donantes glicoconjugados a las mucinas de superficie del parásito (Giorgi and de Lederkremer, 2011). Esta enzima, confiere al parásito la capacidad de cubrir su superficie rápidamente con ácido siálico proveniente de glicoconjugados presentes en el suero o en las superficies celulares. La presencia de ácido siálico en la superficie promueve la digestión de $\mathrm{C} 3$ en un $\mathrm{C} 3 \mathrm{~b}$ inactivo (iC3b), confiriendo resistencia al sistema delcomplemento (Tomlinson et al., 1994). El tratamiento de estos parásitos con sialidasas los hace susceptibles (Kipnis et al., 1981).Algunos trabajos indican que FH se une con mayor afinidad a tripomastigotesmetacíclicos que a epimastigotes(Schenkman et al., 1986). Es probable que esta unión sea a través de sus sitios de unión a ácido siálico. Sin embargo, otros autores afirman que la ineficiente formación de la convertasa de $\mathrm{C} 3$ en la superficie de los tripomastigotes, se debería principalmente a una menor capacidad de unión de factor B a su superficie y no a la mayor unión de FH (Joiner et al., 1986).

\section{Otras moléculas relacionadas}

\subsection{El Mucinas de superficie:}

La superficie de T. cruzi está cubierta de mucinas, glicoproteínas que contribuyen a la protección parasitaria y al establecimiento de una infección persistente. La expresión coordinada de un gran repertorio de mucinas conteniendo regiones variables en los estados parasitarios capaces de infectar a los mamíferos, sugiere una posible estrategia para evadir la respuesta inmune del hospedero (Buscaglia et al., 2006). La superficie de muchos protozoos, incluyendo T. cruzi (Pereira-Chioccola et al., 2000; Schenkman et al., 1993a; Schenkman and Eichinger, 1993; Schenkman et al., 1993b), Cryptosporidiumparvum (Templeton et al., 2004) y el patógeno de peces $T$. carassii(Lischke et al., 2000), están cubiertas de mucinas. Éstas participan en la interfase del parásito con su vector y con su hospedero definitivo, con un doble propósito: proporcionar protección contra el vector $\mathrm{y} / \mathrm{o}$ los mecanismos de defensa derivados del hospedero vertebrado y asegurar el blanco e invasión de tejidos y células específicas (Buscaglia et al., 2006).

Figura 1. Algunas funciones descritas para los SCR de factor $\mathrm{H}$

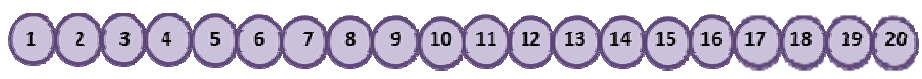

Sitios de unión a C3b

Actividad aceleradora del decaimiento

Actividad co-factora para factor I

Sitio 1 de unión a Herparina

Sitio 2 de unión a Heparina

Sitio 3 de unión a heparina /Ác. siálico

Sitio de unión a CRP 


\begin{tabular}{|c|c|c|}
\hline Proteína & Función & Ubicación o procedencia \\
\hline CRIT & $\begin{array}{l}\text { Se une a C2 y previene su digestión por C1s y } \\
\text { MAPS-2 }\end{array}$ & $\begin{array}{l}\text { Proteína de trans-membrana } \\
\text { expresada en tripomastigotes } \\
\text { de } T . \text { cruzi }\end{array}$ \\
\hline TcCRT & $\begin{array}{l}\text { Se une a } \mathrm{C} 1 \mathrm{q} \text { y } \mathrm{MBL} \text {, inhibiendo la ruta clásica, } \\
\text { y posiblemente, de las lectinas }\end{array}$ & $\begin{array}{l}\text { Intracelular y translocada a la } \\
\text { membrana de tripomastigotes }\end{array}$ \\
\hline Factor $\mathbf{H}$ & $\begin{array}{l}\text { Se une a C3b, acelerando el decaimiento de la } \\
\text { C } 3 \text { convertasa y actuando como cofactor para } \\
\text { factor I. Inhibe la ruta alterna }\end{array}$ & Plasma del hospedero \\
\hline Gp 58/68 & Se une a factor $\mathrm{B}$ y previene su disociación a $\mathrm{Bb}$ & Superficie de tripomastigotes \\
\hline CRP & $\begin{array}{l}\text { Se une a } \mathrm{C} 3 \mathrm{~b} \text { y } \mathrm{C} 4 \mathrm{~b} \text {, provocando una disociación } \\
\text { de la } \mathrm{C} 3 \text { convertasa de la ruta clásica y alterna }\end{array}$ & $\begin{array}{l}\text { Superficie de tripomastigotes, } \\
\text { principalmente, en el flagelo y } \\
\text { bolsillo flagelar }\end{array}$ \\
\hline T-DAF & $\begin{array}{l}\text { Se une a } \mathrm{C} 3 \mathrm{~b} \text { y } \mathrm{C} 4 \mathrm{~b} \text { e inhibe la lisis mediada por } \\
\text { el complemento }\end{array}$ & $\begin{array}{l}\text { Superficie de tripomastigotes } \\
\text { de cultivo, sanguíneos y } \\
\text { metacíclicos }\end{array}$ \\
\hline TS & $\begin{array}{l}\text { Transfieren ácido siálico a la superficie de } \\
\text { tripomastigotes contribuyendo al mimetismo } \\
\text { celular y la posible unión de FH }\end{array}$ & Superficie de tripomastigotes \\
\hline
\end{tabular}

Tabla 1. Proteínas inhibidoras del sistema del complemento utilizadas por T. cruzi.

\section{Conclusiones}

Existen múltiples mecanismos desarrollados por $T$. cruzi para evadir al sistema del complemento del hospedero que actúan a diferentes alturas de esta cascada de eventos. Los principales mecanismos descritos son: 1) la inhibición de la convertasa de C3; 2) la expresión de proteínas protectoras en su superficie, y 3) mimetismo con la célula hospedera. Estos mecanismos están dados por una variada gama de proteínas presentes la superficie del parásito o en el hospedero y que son utilizadas por el parásito en su beneficio. La mayor parte de ellos aún no son bien entendidos y muchos son redundantes. El completo entendimiento de todos ellos y la identificación de otras proteínas relacionadas con estos mecanismos, contribuirá en la búsqueda de mejores blancos terapéuticos y profilácticos para la enfermedad de Chagas.

\section{Referencia}

1. Arlaud, G.J., C. Gaboriaud, N.M. Thielens, M. Budayova-Spano, V. Rossi, and J.C. FontecillaCamps. 2002. Structural biology of the C1 complex of complement unveils the mechanisms of its activation and proteolytic activity. Mol Immunol 39:383-394.

2. Beucher, M., W.S. Meira, V. Zegarra, L.M. Galvao, E. Chiari, and K.A. Norris. 2003. Expression and purification of functional, recombinant Trypanosoma cruzi complement regulatory protein. Protein Expr Purif 27:19-26.

3. Beucher, M., and K.A. Norris. 2008. Sequence diversity of the Trypanosoma cruzi complement regulatory protein family. Infect Immun 76:750758.

4. Buscaglia, C.A., V.A. Campo, A.C. Frasch, and J.M. Di Noia. 2006. Trypanosoma cruzi surface mucins: host-dependent coat diversity. Nat Rev Microbiol 4:229-236. 
5. Cestari, I., I. Evans-Osses, L.J. Schlapbach, I. de Messias-Reason, and M.I. Ramirez. 2012. Mechanisms of complement lectin pathway activation and resistance by trypanosomatid parasites. Mol Immunol 53:328-334.

6. Cestari Idos, S., I. Evans-Osses, J.C. Freitas, J.M. Inal, and M.I. Ramirez. 2008. Complement C2 receptor inhibitor trispanning confers an increased ability to resist complement-mediated lysis in Trypanosoma cruzi. J Infect Dis 198:1276-1283.

7. Cestari Idos, S., A. Krarup, R.B. Sim, J.M. Inal, and M.I. Ramirez. 2009. Role of early lectin pathway activation in the complement-mediated killing of Trypanosoma cruzi. Mol Immunol 47:426-437.

8. Coura, J.R., and J.C. Dias. 2009. Epidemiology, control and surveillance of Chagas disease: 100 years after its discovery. Memorias do Instituto Oswaldo Cruz 104 Suppl 1:31-40.

9. Cross, G.A., and G.B. Takle. 1993. The surface trans-sialidase family of Trypanosoma cruzi. Annu Rev Microbiol 47:385-411.

10. Davis, A.E., 3rd, P. Mejia, and F. Lu. 2008. Biological activities of $\mathrm{C} 1$ inhibitor. Mol Immunol 45:4057-4063.

11. Davitz, M.A., M.G. Low, and V. Nussenzweig. 1986. Release of decay-accelerating factor (DAF) from the cell membrane by phosphatidylinositolspecific phospholipase C (PIPLC). Selective modification of a complement regulatory protein. $J$ Exp Med 163:1150-1161.

12. Ferreira, V., C. Valck, G. Sanchez, A. Gingras, S. Tzima, M.C. Molina, R. Sim, W. Schwaeble, and A. Ferreira. 2004. The classical activation pathway of the human complement system is specifically inhibited by calreticulin from Trypanosoma cruzi. J Immunol 172:3042-3050.

13. Fischer, E., M.A. Ouaissi, P. Velge, J. Cornette, and M.D. Kazatchkine. 1988. gp 58/68, a parasite component that contributes to the escape of the trypomastigote form of $\mathrm{T}$. cruzi from damage by the human alternative complement pathway. Immunology 65:299-303.

14. Giorgi, M.E., and R.M. de Lederkremer. 2011. Trans-sialidase and mucins of Trypanosoma cruzi: an important interplay for the parasite. Carbohydr Res 346:1389-1393.

15. Haberland, A., S.G. Saravia, G. Wallukat, R. Ziebig, and I. Schimke. 2012. Chronic Chagas disease: from basics to laboratory medicine. Clin Chem Lab Med 0:1-24.

16. Hourcade, D., V.M. Holers, and J.P. Atkinson. 1989. The regulators of complement activation (RCA) gene cluster. Adv Immunol 45:381-416.
17. Huang, Y., F. Qiao, R. Abagyan, S. Hazard, and S. Tomlinson. 2006. Defining the CD59-C9 binding interaction. J Biol Chem 281:27398-27404.

18. Inal, J.M. 2005. Complement C2 receptor inhibitor trispanning: from man to schistosome. Springer Semin Immunopathol 27:320-331.

19. Inal, J.M., and J.A. Schifferli. 2002. Complement C2 receptor inhibitor trispanning and the betachain of $\mathrm{C} 4$ share a binding site for complement C2. J Immunol 168:5213-5221.

20. Johnson, C.A., Y.Y. Kleshchenko, A.O. Ikejiani, A.N. Udoko, T.C. Cardenas, S. Pratap, M.A. Duquette, M.F. Lima, J. Lawler, F. Villalta, and P.N. Nde. 2012. Thrombospondin-1 interacts with Trypanosoma cruzi surface calreticulin to enhance cellular infection. PLoS One 7:e40614.

21. Joiner, K., A. Sher, T. Gaither, and C. Hammer. 1986. Evasion of alternative complement pathway by Trypanosoma cruzi results from inefficient binding of factor B. Proc Natl Acad Sci US A 83:6593-6597.

22. Kemper, C., J.P. Atkinson, and D.E. Hourcade. 2009. Properdin: Emerging Roles of a PatternRecognition Molecule. Annu Rev Immunol

23. Kemper, C., J.P. Atkinson, and D.E. Hourcade. 2010. Properdin: emerging roles of a patternrecognition molecule. Annu Rev Immunol 28:131155.

24. Kinoshita, T., M.E. Medof, and V. Nussenzweig. 1986. Endogenous association of decayaccelerating factor (DAF) with $\mathrm{C} 4 \mathrm{~b}$ and $\mathrm{C} 3 \mathrm{~b}$ on cell membranes. J Immunol 136:3390-3395.

25. Kipnis, T.L., J.R. David, C.A. Alper, A. Sher, and W.D. da Silva. 1981. Enzymatic treatment transforms trypomastigotes of Trypanosoma cruzi into activators of alternative complement pathway and potentiates their uptake by macrophages. Proc Natl Acad Sci U S A 78:602-605.

26. Lambris, J.D., D. Ricklin, and B.V. Geisbrecht. 2008. Complement evasion by human pathogens. Nat Rev Microbiol 6:132-142.

27. Lepow, I.H., G.B. Naff, E.W. Todd, J. Pensky, and C.F. Hinz. 1963. Chromatographic resolution of the first component of human complement into three activities. J Exp Med 117:983-1008.

28. Lischke, A., C. Klein, Y.D. Stierhof, M. Hempel, A. Mehlert, I.C. Almeida, M.A. Ferguson, and P. Overath. 2000. Isolation and characterization of glycosylphosphatidylinositol-anchored, mucin-like surface glycoproteins from bloodstream forms of the freshwater-fish parasite Trypanosoma carassii. Biochem J 345 Pt 3:693-700.

29. Liszewski, M.K., and J.P. Atkinson. 1996. Membrane cofactor protein (MCP; CD46). 
Isoforms differ in protection against the classical pathway of complement. J Immunol 156:44154421.

30. Macedo, A.M., R.P. Oliveira, and S.D. Pena. 2002. Chagas disease: role of parasite genetic variation in pathogenesis. Expert Rev Mol Med 4:1-16.

31. Mortara, R.A., W.K. Andreoli, N.N. Taniwaki, A.B. Fernandes, C.V. Silva, M.C. Fernandes, C. L'Abbate, and S. Silva. 2005. Mammalian cell invasion and intracellular trafficking by Trypanosoma cruzi infective forms. An Acad Bras Cienc 77:77-94.

32. Naresha, S., A. Suryawanshi, M. Agarwal, B.P. Singh, and P. Joshi. 2009. Mapping the complement $\mathrm{Clq}$ binding site in Haemonchus contortus calreticulin. Mol Biochem Parasitol $166: 42-46$

33. Nogueira, N., C. Bianco, and Z. Cohn. 1975. Studies on the selective lysis and purification of Trypanosoma cruzi. J Exp Med 142:224-229.

34. Norris, K.A. 1998. Stable transfection of Trypanosoma cruzi epimastigotes with the trypomastigote-specific complement regulatory protein cDNA confers complement resistance. Infect Immun 66:2460-2465.

35. Norris, K.A., B. Bradt, N.R. Cooper, and M. So. 1991. Characterization of a Trypanosoma cruzi C3 binding protein with functional and genetic similarities to the human complement regulatory protein, decay-accelerating factor. $J$ Immunol 147:2240-2247.

36. Norris, K.A., and J.E. Schrimpf. 1994. Biochemical analysis of the membrane and soluble forms of the complement regulatory protein of Trypanosoma cruzi. Infect Immun 62:236-243.

37. Oladiran, A., and M. Belosevic. 2009. Trypanosoma carassii calreticulin binds host complement component $\mathrm{C} 1 \mathrm{q}$ and inhibits classical complement pathway-mediated lysis. Dev Comp Immunol

38. Osorio, L., I. Rios, B. Gutierrez, and J. Gonzalez. 2012. Virulence factors of Trypanosoma cruzi: who is who? Microbes Infect

39. Pangburn, M.K. 2000. Host recognition and target differentiation by factor $\mathrm{H}$, a regulator of the alternative pathway of complement. Immunopharmacology 49:149-157.

40. Pereira-Chioccola, V.L., A. Acosta-Serrano, I. Correia de Almeida, M.A. Ferguson, T. SoutoPadron, M.M. Rodrigues, L.R. Travassos, and S. Schenkman. 2000. Mucin-like molecules form a negatively charged coat that protects Trypanosoma cruzi trypomastigotes from killing by human anti- alpha-galactosyl antibodies. J Cell Sci 113 ( Pt 7):1299-1307.

41. Ramirez, G., C. Valck, L. Aguilar, U. Kemmerling, R. Lopez-Munoz, G. Cabrera, A. Morello, J. Ferreira, J.D. Maya, N. Galanti, and A. Ferreira. 2012. Roles of Trypanosoma cruzi calreticulin in parasite-host interactions and in tumor growth. Mol Immunol 52:133-140.

42. Ramirez, G., C. Valck, M.C. Molina, C.H. Ribeiro, N. Lopez, G. Sanchez, V.P. Ferreira, R. Billetta, L. Aguilar, I. Maldonado, P. Cattan, W. Schwaeble, and A. Ferreira. 2011. Trypanosoma cruzi calreticulin: a novel virulence factor that binds complement $\mathrm{C} 1$ on the parasite surface and promotes infectivity. Immunobiology 216:265-273.

43. Rimoldi, M.T., A.J. Tenner, D.A. Bobak, and K.A. Joiner. 1989. Complement component C1q enhances invasion of human mononuclear phagocytes and fibroblasts by Trypanosoma cruzi trypomastigotes. J Clin Invest 84:1982-1989.

44. Schenkman, R.P., F. Vandekerckhove, and S. Schenkman. 1993a. Mammalian cell sialic acid enhances invasion by Trypanosoma cruzi. Infect Immun 61:898-902.

45. Schenkman, S., and D. Eichinger. 1993. Trypanosoma cruzi trans-sialidase and cell invasion. Parasitol Today 9:218-222.

46. Schenkman, S., M.A. Ferguson, N. Heise, M.L. de Almeida, R.A. Mortara, and N. Yoshida. 1993b. Mucin-like glycoproteins linked to the membrane by glycosylphosphatidylinositol anchor are the major acceptors of sialic acid in a reaction catalyzed by trans-sialidase in metacyclic forms of Trypanosoma cruzi. Mol Biochem Parasitol 59:293-303.

47. Schenkman, S., M.L. Guther, and N. Yoshida. 1986. Mechanism of resistance to lysis by the alternative complement pathway in Trypanosoma cruzi trypomastigotes: effect of specific monoclonal antibody. J Immunol 137:1623-1628.

48. Sjoberg, A.P., L.A. Trouw, and A.M. Blom. 2009. Complement activation and inhibition: a delicate balance. Trends Immunol 30:83-90.

49. Solana, M.E., M.F. Ferrer, M.M. Novoa, W.C. Song, and R.M. Gomez. 2012. Decay-accelerating factor 1 deficiency exacerbates Trypanosoma cruzi-induced murine chronic myositis. Muscle Nerve 46:582-587.

50. Suchitra, S., K.A. Anbu, D.K. Rathore, M. Mahawar, B.P. Singh, and P. Joshi. 2008. Haemonchus contortus calreticulin binds to $\mathrm{C}$ reactive protein of its host, a novel survival strategy of the parasite. Parasite Immunol 30:371374. 
51. Suchitra, S., and P. Joshi. 2005. Characterization of Haemonchus contortus calreticulin suggests its role in feeding and immune evasion by the parasite. Biochim Biophys Acta 1722:293-303.

52. Tambourgi, D.V., R.A. Cavinato, C.M. De Abreu, B.A. Peres, and T.L. Kipnis. 1995. Detection of Trypanosoma-decay accelerating factor antibodies in mice and humans infected with Trypanosoma cruzi. Am J Trop Med Hyg 52:516-520.

53. Tambourgi, D.V., T.L. Kipnis, W.D. da Silva, K.A. Joiner, A. Sher, S. Heath, B.F. Hall, and G.B. Ogden. 1993. A partial cDNA clone of trypomastigote decay-accelerating factor (T-DAF), a developmentally regulated complement inhibitor of Trypanosoma cruzi, has genetic and functional similarities to the human complement inhibitor DAF. Infect Immun 61:3656-3663.

54. Templeton, T.J., C.A. Lancto, V. Vigdorovich, C. Liu, N.R. London, K.Z. Hadsall, and M.S. Abrahamsen. 2004. The Cryptosporidium oocyst wall protein is a member of a multigene family and has a homolog in Toxoplasma. Infect Immun 72:980-987.

55. Tomlinson, S., L.C. Pontes de Carvalho, F. Vandekerckhove, and V. Nussenzweig. 1994. Role of sialic acid in the resistance of Trypanosoma cruzi trypomastigotes to complement. J Immunol 153:3141-3147.

56. Valck, C., G. Ramirez, N. Lopez, C.H. Ribeiro, I. Maldonado, G. Sanchez, V.P. Ferreira, W. Schwaeble, and A. Ferreira. 2010. Molecular mechanisms involved in the inactivation of the first component of human complement by Trypanosoma cruzi calreticulin. Mol Immunol 47:1516-1521.

57. Van Voorhis, W.C., L. Barrett, R. Koelling, and A.G. Farr. 1993. FL-160 proteins of Trypanosoma cruzi are expressed from a multigene family and contain two distinct epitopes that mimic nervous tissues. J Exp Med 178:681-694.

58. Velge, P., M.A. Ouaissi, J. Cornette, D. Afchain, and A. Capron. 1988. Identification and isolation of Trypanosoma cruzi trypomastigote collagenbinding proteins: possible role in cell-parasite interaction. Parasitology 97 ( Pt 2):255-268.

59. Wallis, R., D.A. Mitchell, R. Schmid, W.J. Schwaeble, and A.H. Keeble. 2010. Paths reunited: Initiation of the classical and lectin pathways of complement activation. Immunobiology 215:1-11. 